\title{
Chronic Intermittent Materno-Fetal Hyperoxygenation in Late Gestation May Improve on Hypoplastic Cardiovascular Structures Associated with Cardiac Malformations in Human Fetuses
}

\author{
Thomas Kohl
}

Received: 22 July 2009/ Accepted: 4 November 2009/Published online: 19 December 2009

(C) The Author(s) 2009. This article is published with open access at Springerlink.com

\begin{abstract}
Hypoplasia of cardiovascular structures is a common finding in fetuses with cardiac malformations. Materno-fetal hyperoxygenation (HO) during late gestation promotes venous return to the fetal heart. This analysis in human fetuses sought to define whether this "loading" effect might improve hypoplastic cardiovascular dimensions. Fifteen late-gestation fetuses presented with varying degrees of hypoplastic cardiovascular structures. In these cases, chronic intermittent materno-fetal HO was administered during periods ranging from 8 to 33 days. Cardiac measurements were taken before and at the end of treatment and translated into $Z$-scores as well as plotted on normal growth charts. During the treatment period, chronic intermittent materno-fetal HO was associated with improved dimensions of $\geq 1$ hypoplastic cardiovascular structures in most fetuses. However, in some cases, the effect of HO was neutralized or impaired by the presence of ventricular septal defects as well as obstructions to ventricular filling or emptying. Chronic intermittent materno-fetal HO near term may be associated with improvements of hypoplastic cardiovascular dimensions in fetuses with a spectrum of cardiac malformations. This effect may facilitate postnatal treatment and improve prognosis in suitable cases.
\end{abstract}

This manuscript is cordially dedicated to Ulrich Gembruch, Director of the Department of Obstetrics and Prenatal Medicine, University of Bonn.

T. Kohl $(\bowtie)$

German Center for Fetal Surgery and Minimally Invasive

Therapy, University Hospital of Bonn, Bonn 53105, Germany

e-mail: thomas.kohl@ukb.uni-bonn.de
Keywords Coarctation - Congenital heart disease . Fetal cardiac intervention - Fetus - Hypoplastic left heart complex · Hyperoxygenation - Left heart hypoplasia . Right heart hypoplasia

\section{Introduction}

Hypoplasia of one or several cardiovascular structures is a common feature of many cardiac malformations detected during fetal life. Based on hypoplasia location and severity, this finding may bear important and life-long consequences for postnatal treatment and prognosis. Materno-fetal hyperoxygenation $(\mathrm{HO})$ in the third trimester results in fetal pulmonary vasodilation [8] and increased venous return to the fetal heart. Similar to findings in chicks, in which increased ventricular preload is compensated by myocyte proliferation in hypoplastic left ventricles [1], the resulting increase in cardiac preload from pulmonary vasodilation might, with time, also improve hypoplastic fetal cardiovascular dimensions in humans. The purpose of this report is to describe the impact of chronic intermittent maternofetal $\mathrm{HO}$ on the dimensions of hypoplastic cardiovascular structures in 15 human fetuses with a variety of cardiac malformations.

\section{Patients and Methods}

The studies were performed in 15 pregnant women and their fetuses between 33 weeks +5 days and 38 weeks +0 days of gestation; all fetuses had normal karyotypes. Thirteen of the 15 fetuses had $\geq 1$ hypoplastic left heart structures; of these, 1 fetus had a severely hypoplastic tricuspid valve and right ventricle associated with anatomically 


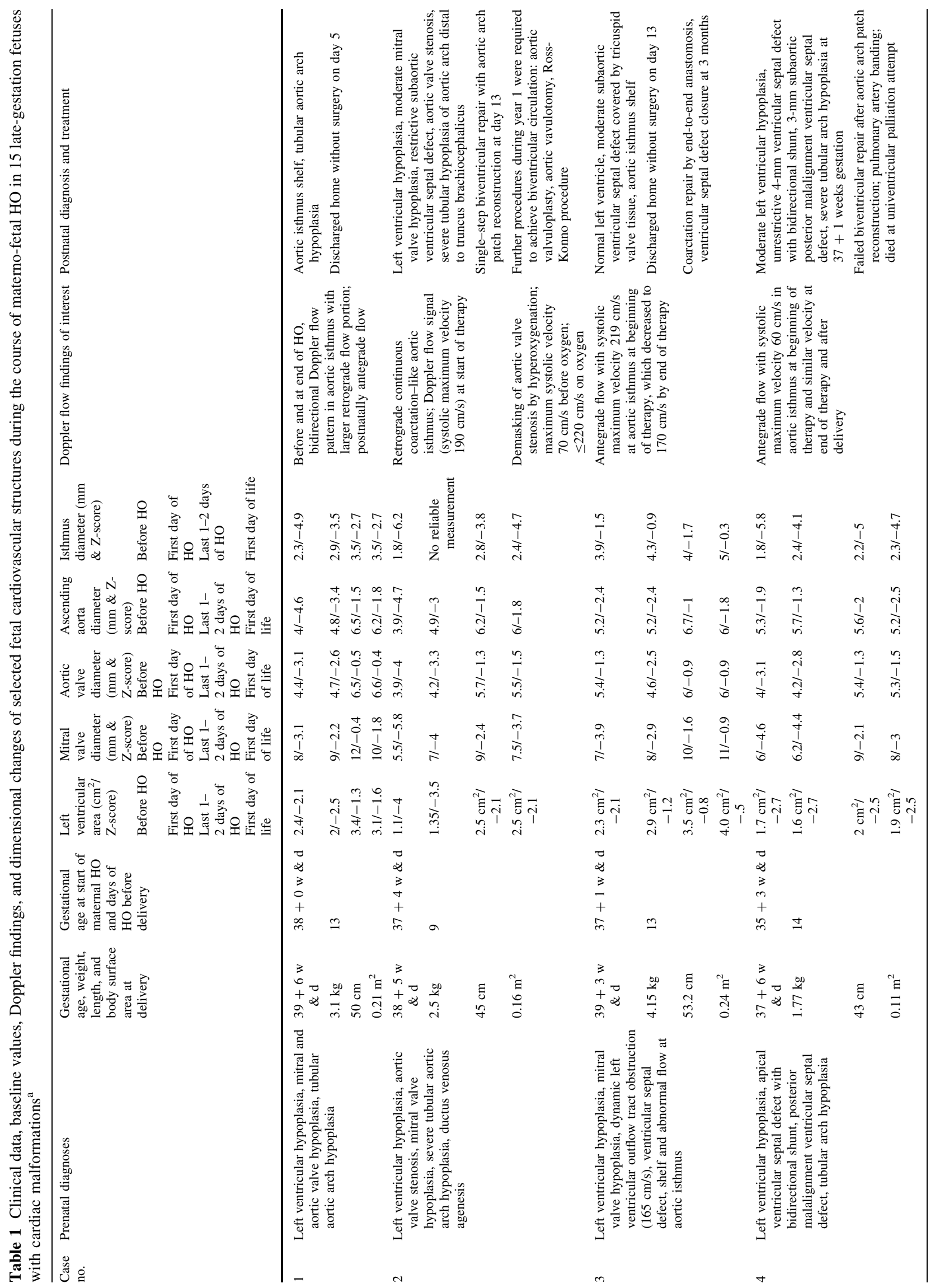




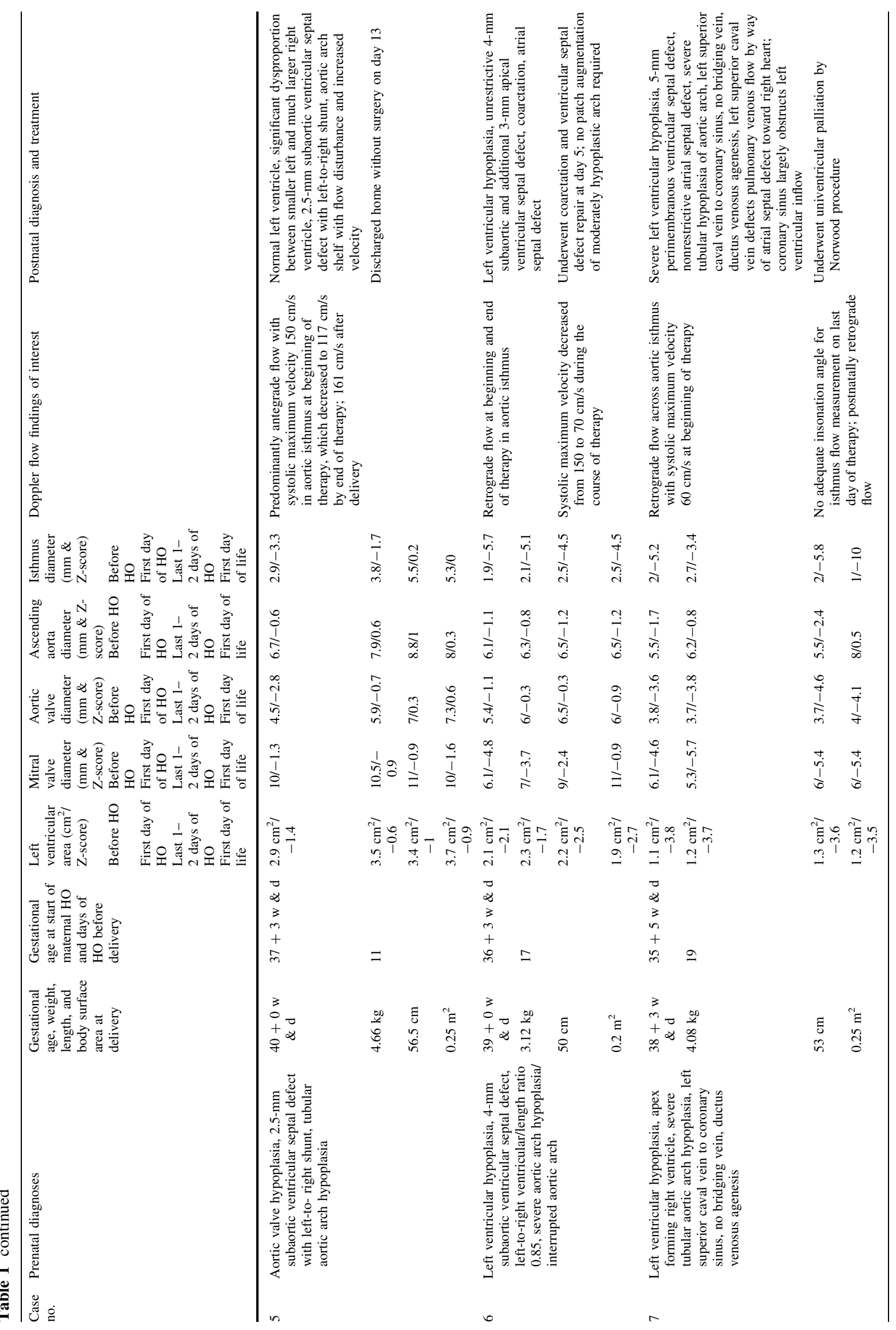




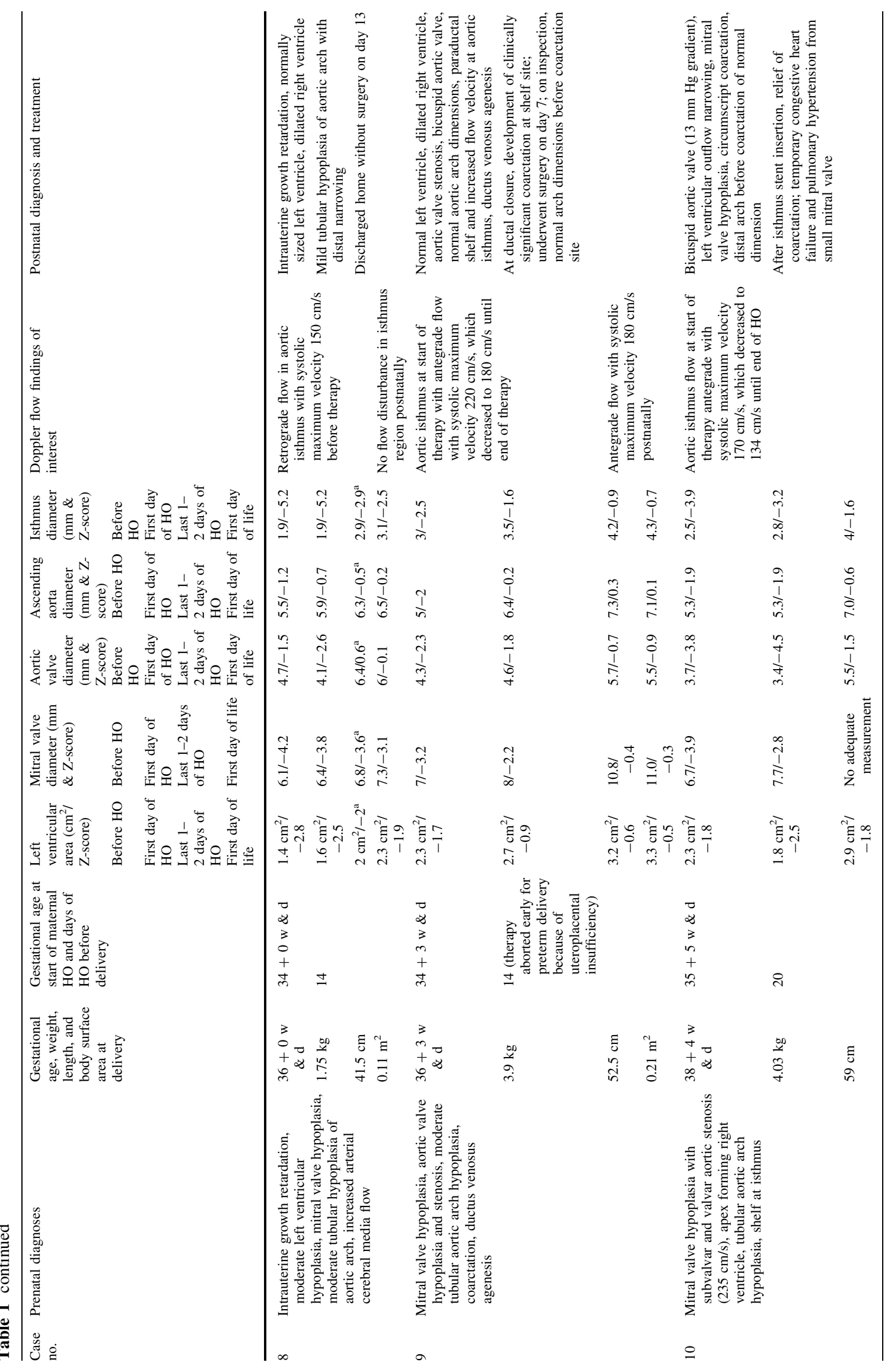




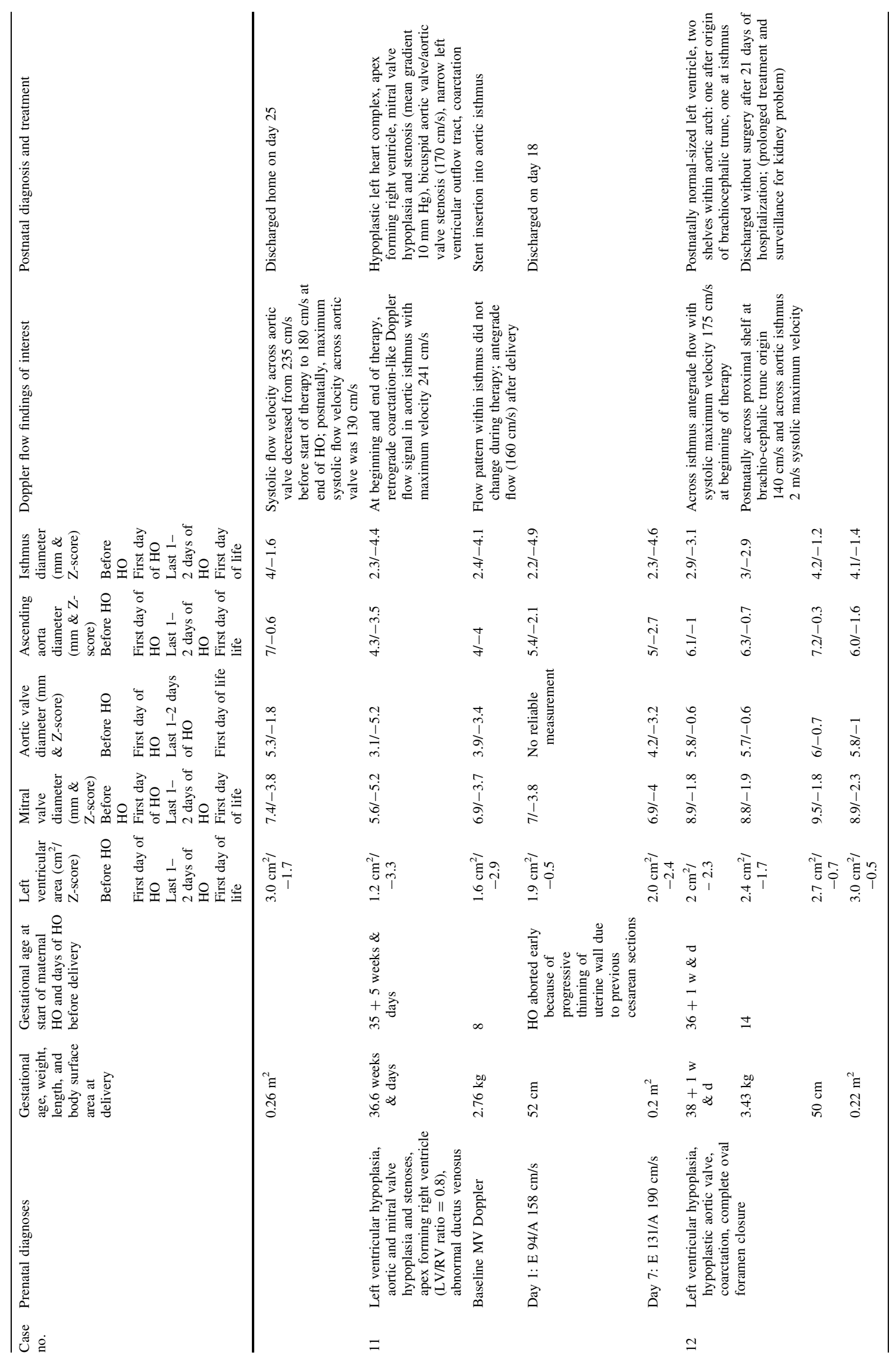




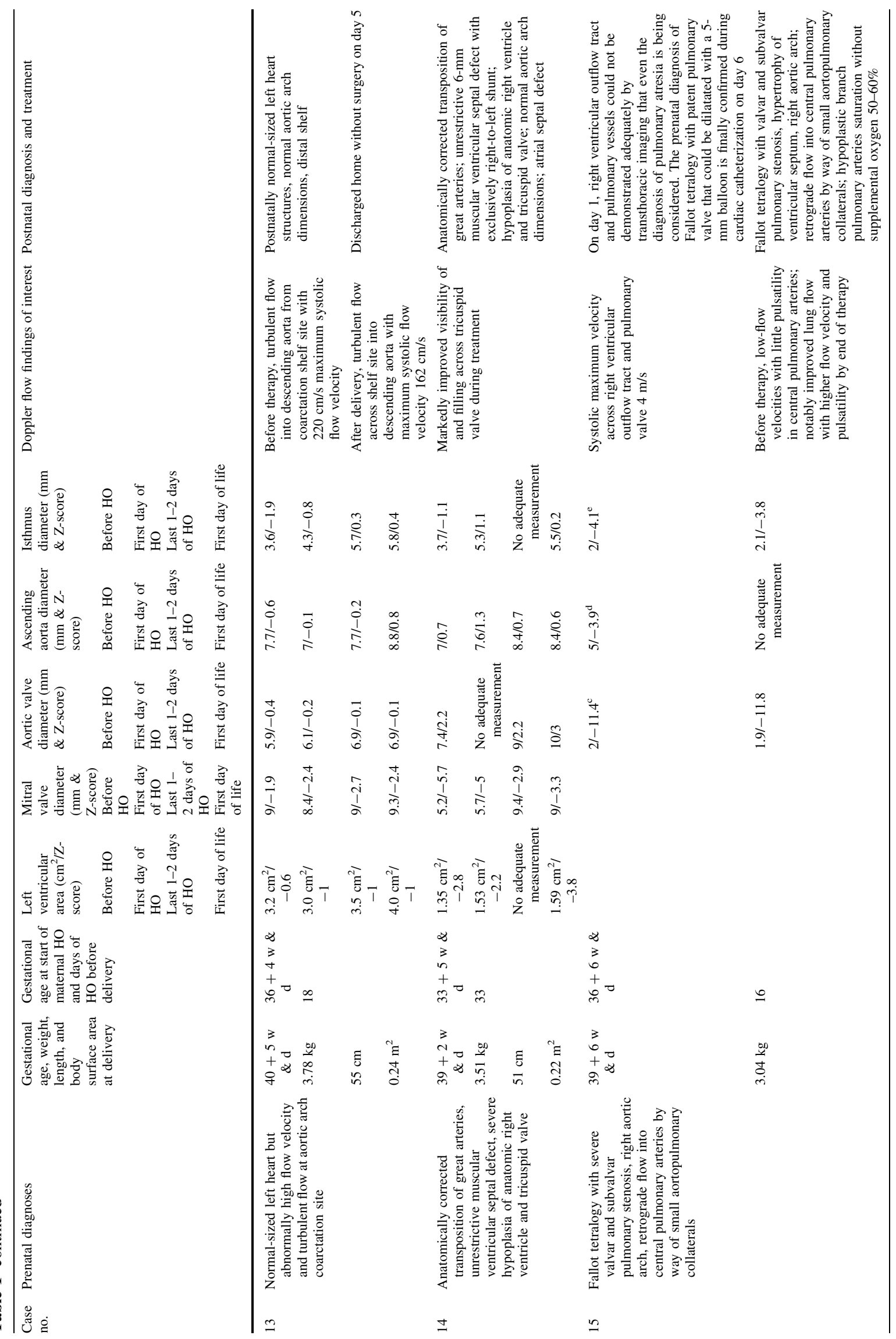




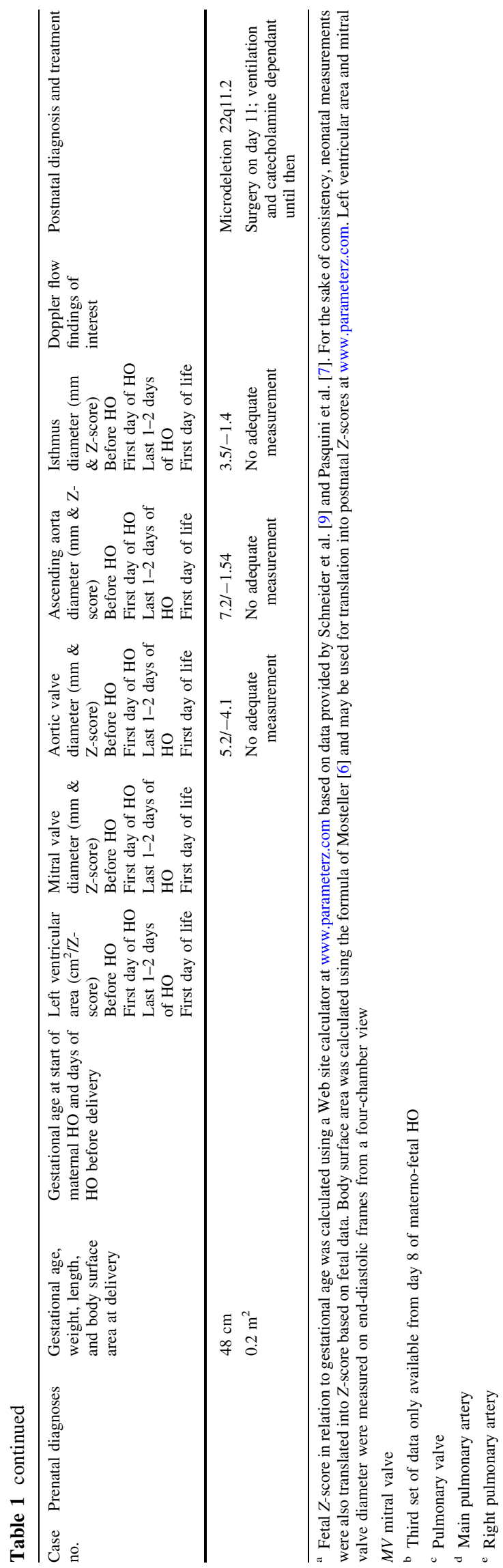

corrected transposition of the great arteries, and another had markedly hypoplastic pulmonary vessels and lung blood flow associated with tetralogy of Fallot (Table 1).

\section{Informed Consent and Treatment Protocol}

Based on fetal echocardiographic findings, the 15 expectant mothers were offered chronic intermittent materno-fetal HO during the final weeks of gestation. The individual procedures were performed after receiving approval by the heads of the local committee on human research and maternal informed consent.

On the first day, the women underwent a detailled multimodal prenatal sonogram. Then they were requested to wear a rebreathing oxygen mask. The oxygen flow was adjusted to a maternal arterial $P_{\mathrm{O}_{2}}$ between 250 and $300 \mathrm{~mm} \mathrm{Hg}$ by ultrasound-guided puncture of the maternal femoral artery using a 26-gauge needle.

On the first day, oxygen was provided for approximately 6 hours. After 1 to 2 hours of materno-fetal HO, the response of the pulmonary vascular circulation and the loading effect of increased venous return to both ventricles on fetal hemodynamics were assessed by detailled sonography as previously outlined.

From the second day onward, for a total between 8 and 33 days (mean 15.5 and median 14), the women underwent $3 \times 4 \mathrm{~h} \mathrm{HO} / \mathrm{d}$ until delivery. The timing and length of materno-fetal $\mathrm{HO}$ were determined by gestational age at referral, response to therapy, and obstetric considerations. No attempts were made to prolong gestation in cases for whom spontaneous delivery ensued.

The fetuses were monitored by fetal echocardiography and cardiotocography and the expectant mothers by daily clinical examination and interrogation. After delivery, the mothers were requested to undergo chest X-ray and pulmonary function test to monitor for maternal pulmonary morbidity from the experimental treatment approach.

\section{Measurements}

Multimodal fetal echocardiography was performed using a high-end ultrasound system (Acuson Sequoia 512; AcusonA-Siemens-Company, Erlangen, Germany). Measurements of ventricular area as well as atrioventricular valve diameter were taken in the four-chamber plane at end-diastole. Semilunar valve diameter was measured during ventricular ejection. In addition, the largest diameter of great vessel regions of interest were measured during early systole.

Fetal cardiovascular flow regions of interest were assessed by color and pulsed Doppler echocardiography at insonation angles $<20^{\circ}$. Before and during materno-fetal 
Table 2 The effect of materno-fetal HO on fetal left heart filling and emptying velocities ${ }^{\mathrm{a}}$

\begin{tabular}{|c|c|c|c|c|c|}
\hline \multirow{2}{*}{\multicolumn{3}{|c|}{ Measurements provided as }} & \multicolumn{3}{|c|}{ Baseline/during $\mathrm{HO}$} \\
\hline & & & \multicolumn{3}{|c|}{$\left(21 \% \mathrm{O}_{2}\right) /\left(\right.$ maternal $\left.\mathrm{Fi}_{\mathrm{O}_{2}} 40-50 \%\right)$} \\
\hline Case no. & MV-E (cm/s) & MV-A (cm/s) & $\mathrm{AoV}(\mathrm{cm} / \mathrm{s})$ & $\begin{array}{l}\text { Pulmonary vein } \\
\text { (mean velocity }[\mathrm{cm} / \mathrm{s}] \text { ) }\end{array}$ & $\begin{array}{l}\text { Color Doppler } \\
\text { (lung flow increase) }\end{array}$ \\
\hline 1 & $40 / 50$ & $55 / 53$ & No data & $16 / 31$ & ++ to +++ \\
\hline 2 & $45 / 47$ & $66 / 67$ & $62 / 200$ & $27 / 36$ & + to +++ \\
\hline 3 & $49 /-$ & $43 / 72$ & $165 / 123$ & $24 / *$ & + to ++ \\
\hline 4 & $34 / 34$ & $46 / 64$ & $81 / 77$ & $14 / 19$ & + to ++ \\
\hline 5 & $56 / 50$ & $64 / 80$ & $82 / 101$ & $34 / 39$ & ++ to +++ \\
\hline 6 & $29 / 53$ & $35 / 61$ & $110 / 124$ & $13 / 20$ & + to ++ \\
\hline 7 & $52 / 74$ & $76 / 74$ & $92 / 95$ & $14 / *$ & ++ to +++ \\
\hline 8 & $56 / 42$ & $49 / 33$ & $85 / 73$ & $24 / 37$ & + to +++ \\
\hline 9 & $46 /^{b}$ & $61 /^{\mathrm{b}}$ & $206 / 234$ & $21 /^{b}$ & + to ++ \\
\hline 10 & $60 / 66$ & $81 / 78$ & $235 / 216$ & $29 / 29$ & ++ to +++ \\
\hline 11 & $94 /^{\mathrm{b}}$ & $158 / 166$ & $91 / 110$ & $22 / 22$ & + to ++ \\
\hline 12 & $\mathrm{~b} / 30$ & $\mathrm{~b} / 73$ & $\mathrm{~b} / 94$ & $15 / 20$ & + to ++ \\
\hline 13 & $38 / 46$ & $46 / 55$ & $70 / 84$ & $\mathrm{~b} / \mathrm{b}$ & + to ++ \\
\hline 14 & $50 / 54$ & $56 / 78$ & $103 /^{\mathrm{b}}$ & $14 / 22$ & + to ++ \\
\hline 15 & No data & No data & No data & $18 / 25$ & + to ++ \\
\hline Available pairs & $10 / 15$ & $12 / 15$ & $11 / 15$ & $11 / 15$ & $15 / 15$ \\
\hline Flow increase & $7 / 10$ & $8 / 12$ & $7 / 11$ & $9 / 11$ & $15 / 15$ \\
\hline Flow decrease & $2 / 10$ & $4 / 12$ & $4 / 11$ & - & - \\
\hline No change & $1 / 10$ & - & - & $2 / 10$ & - \\
\hline$p$ & 0.16 & 0.05 & 0.32 & $<0.0007$ & $<0.0001$ \\
\hline
\end{tabular}

$(+$ to +++$)=$ quantitative assessment of color Doppler lung flow changes on motion files in the four-chamber view: $+=$ venous flow Doppler signal most visible within inner third, $++=$ inner and middle third, and $(+++)$ inner to outer third of the region between the posterior wall of the left atrium and the chest wall. $F i_{O_{2}}$ fraction of inspired oxygen, $M V-E$ mitral valve early diastolic filling velocity, $M V$ - $A$ mitral valve late diastolic filling velocity, AoV aortic valve velocity

${ }^{a}$ Measurements were taken before (baseline) and during the first hour of materno-fetal hyperoxygenation (HO) on the first day of treatment

b Inadequate Doppler measurement due to breathing, inadequate insonation angle, or poor imaging quality

HO on the first treatment day, fetal pulmonary venous blood flow mean velocity as well as quantitative changes in color Doppler signal of the lung vessels were compared from still and motion files, respectively, to document pulmonary vasodilation (Table 2).

Measurements of fetal cardiovascular structures of interest were taken at four time points (Table 1). The first and second sets of measurements were taken on the first treatment day right before and during the first hours of materno-fetal HO to document whether oxygeninduced fetal pulmonary vasodilation does in fact load the fetal heart. The third set of measurements represents fetal cardiovascular dimensions taken during the last 2 days of materno-fetal HO. The fourth set of measurements was taken by pediatric cardiologists blinded to the prenatal measurements after delivery during the first 2 days of life.

\section{Data Presentation and Analysis}

To compare changes in cardiovascular dimensions of the hyperoxygenated fetuses with normal ones, the data were expressed as gestational age-related Z-scores based on data provided by Schneider et al. [9] and Pasquini et al. [7] employing a Web site calculator available at http://www. parameterz.com. In addition, for enhanced visual demonstration, selected cardiovascular dimensions of the 13 fetuses with hypoplastic left heart structures were plotted on fetal cardiac growth curves for normals provided by Schneider et al. [9] and Matsui et al. [5].

Statistical analysis on fetal cardiac dimensions, atrioventricular and semilunar valve flows, and central pulmonary vein mean flow velocities during loading of the fetal heart was performed using paired Student $t$ test (Table 2). In addition, the same test was performed on 

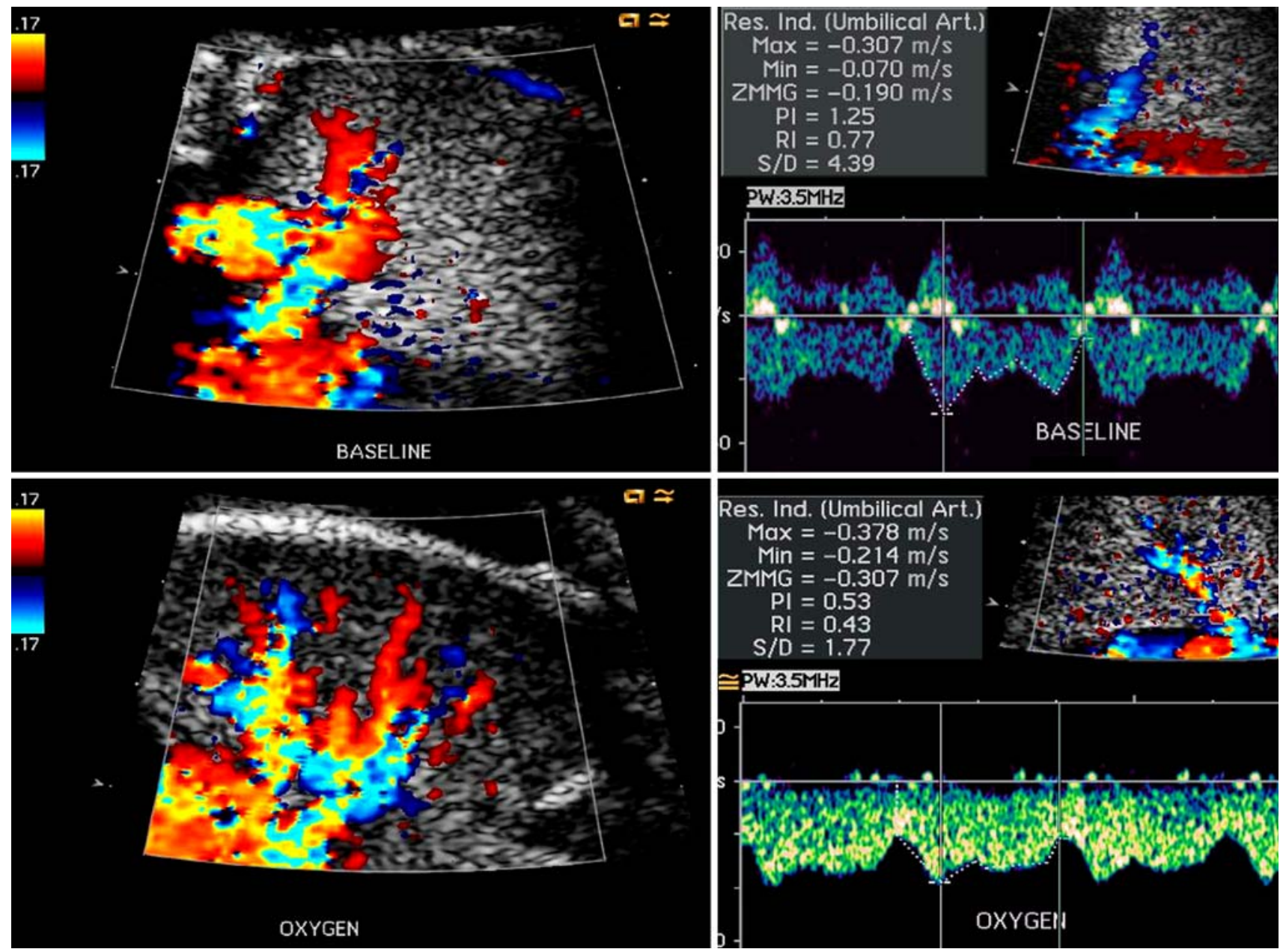

Fig. 1 Typical effect of materno-fetal $\mathrm{HO}$ on fetal lung blood flow as demonstrated by color and pulsed Doppler echocardiography in a fetus with hypoplasia of left heart cardiovascular structures. Within minutes of initiating treatment, materno-fetal $\mathrm{HO}$ results in marked

semiquantitative data derived from color Doppler motion files of fetal lung flow before and during materno-fetal $\mathrm{HO}$, and $p \leq 0.05$ was considered statistically significant. The study addressed the following questions:

1. Is chronic intermittent materno-fetal $\mathrm{HO}$ - by increasing fetal pulmonary venous return and, hence, cardiac preload-associated with an increase in fetal hypoplastic cardiovascular dimensions?

2. Are there any adverse maternal events during and after materno-fetal $\mathrm{HO}$ ?

3. Are there any adverse fetal or neonatal complications?

\section{Results}

Within the first $2 \mathrm{~h}$ of initiating treatment, materno-fetal HO resulted in statistically significant increases of color Doppler changes and increases in pulmonary venous flow velocity (Color and pulsed Doppler frames before (top panel) and during (bottom panel) materno-fetal HO). (Color figure online)

pulmonary venous mean flow velocity $(p=0.0007)$ and mitral valve late diastolic ("a-wave") flow velocity $(p=0.05)$ (Table 1 and Fig. 1). During this left heart loading phase, significant increases in the dimensions of the mitral valve $(p=0.02)$, ascending aorta $(p=0.03)$, and aortic isthmus $(p=0.03)$ were observed.

The following changes in fetal cardiac dimensions and the following changes in $Z$-scores were observed in the fetuses during the course of chronic intermittent materno-fetal HO between 8 and 33 days (mean 15.5 and median 14) (Table 1 and Figs. 2-4).

\section{Ventricular Area}

Changes in areas and Z-scores of left ventricles $(n=13)$, as well as a left-sided right ventricle $(n=1)$, were available in 14 of 15 fetuses. In 7 of these fetuses, marked area 


\begin{tabular}{|lllll|}
\hline \multirow{3}{*}{ Patients } & $1=\mathbf{\Delta}$ & $4=\boldsymbol{D}$ & $7=\diamond$ & $10=0$ \\
& $2=\boldsymbol{\Delta}$ & $5=x$ & $8=\S$ & $11=x$ \\
& $3=$ & $6=0$ & $9=\star$ & $12=0$
\end{tabular}

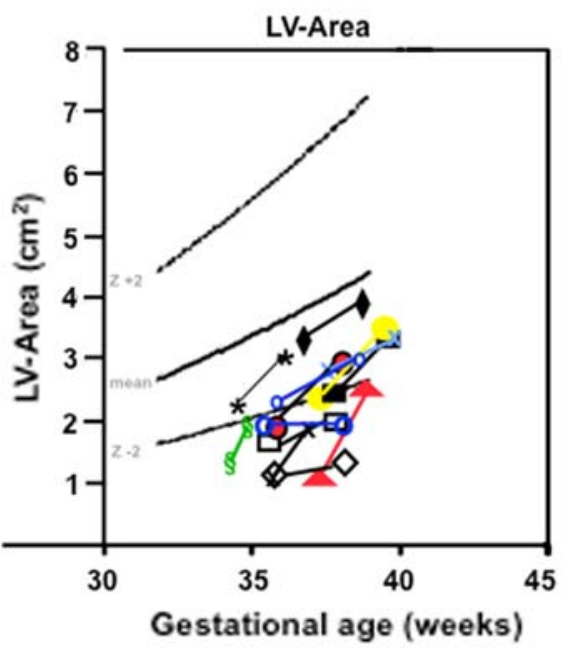

ig. 2 Dimensions of left ventricular area and mitral valve diameter in the four-chamber plane before and at the end of materno-fetal $\mathrm{HO}$ in 13 fetuses with cardiovascular hypoplasia of $\geq 1$ left heart structures. Black solid lines indicate mean values $\pm 2 z$-based on

and Z-score increases for left ventricular area were observed in the cardiac four-chamber view (Fig. 2) during the course of therapy. In five fetuses, the Z-scores for left ventricular area developed in parallel to the growth curve of normals. In one fetus, the $Z$-score for the left ventricular area, and in one fetus, the $Z$-score for the right ventricular area decreased progressively during the course of therapy.

\section{Atrioventricular Valve Diameter}

Changes in diameters and Z-scores of the left-sided mitral or tricuspid valves were available in 14 of 15 fetuses. In 9 of these fetuses, marked increases of dimension and $Z$ scores of mitral valve $(n=8)$ or tricuspid valve $(n=1)$ diameter were observed during the treatment period (Fig. 2). In two fetuses, the mitral valve diameter developed in parallel to the growth curve of normals; in three fetuses, the mitral valve diameter remained unchanged, hence decreasing progressively below the lower limit of normal during the course of therapy. data provided by Schneider et al. [9]. Black dots to the right of the figure are provided to facilitate the comprehension of even small dimensional changes on flow

\section{Semilunar Valve Diameter}

Changes in diameters and Z-scores of aortic $(n=13)$ or pulmonary valves $(n=1)$ were available in 14 of 15 fetuses. In 10 of these fetuses, marked diameter and Zscore increases were observed (Fig. 3) during the course of therapy. In two fetuses, the aortic valve diameter developed in parallel to the growth curve of normals. In two fetuses, the dimensions of the aortic valve remained unchanged such that their Z-scores decreased progressively during the course of therapy.

\section{Great Artery Diameter}

Changes in diameters and $Z$-scores of the ascending aorta $(n=14)$ or main pulmonary artery $(n=1)$ were available from all fetuses. In 10 of these fetuses, marked diameter and $Z$-score increases of the ascending aorta $(n=19)$ or main pulmonary artery $(n=1)$ were observed (Fig. 3) during the course of therapy. In three fetuses, the ascending aorta diameter developed parallel to the growth curve of 

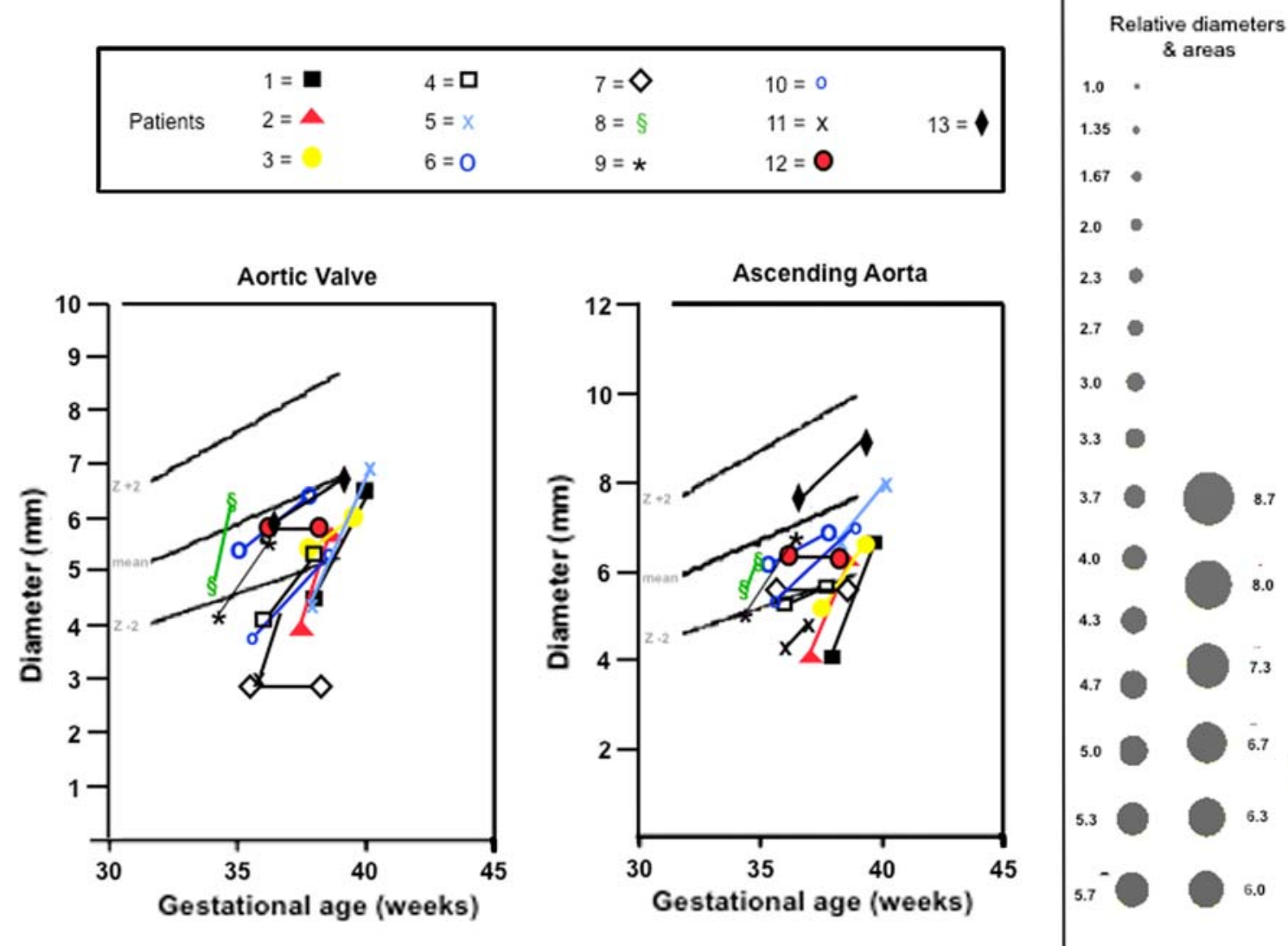

Fig. 3 Diameters of the aortic valve and ascending aorta in the fourchamber plane before and at the end of materno-fetal $\mathrm{HO}$ in fetuses

lines indicate mean values $\pm 2 z$-based on data provided by Schneider et al. [9]. Black dots to the right of the figure are provided to facilitate the comprehension of even small dimensional changes on flow

with cardiovascular hypoplasia of $\geq 1$ left heart structures. Black solid

Fig. 4 Diameters and Z-scores of the aortic isthmus in the fourchamber plane before and at the end of materno-fetal $\mathrm{HO}$ in fetuses with cardiovascular hypoplasia of $\geq 1$ left heart structures. Z-scores for normals are based on data provided by Matsui et al. [5]. Black dots to the right of the figure are provided to facilitate the comprehension of even small dimensional changes on flow
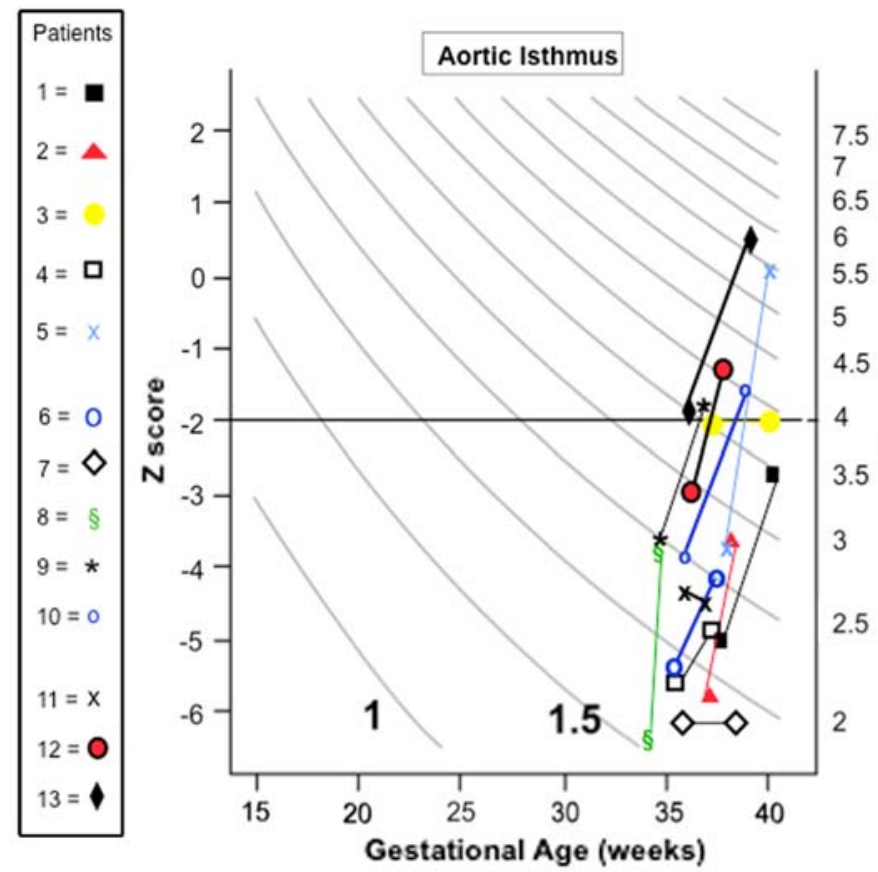

Relative diameters $\&$ areas

$1.0 \quad-$

$1.35 \bullet$

$1.67 \bullet$

2.0

2.3

2.7

3.0

3.3

3.7

4.0

4.3

4.7

5.0

5.3

5.7 
normals. In two fetuses, the diameter of the ascending aorta remained unchanged such that their $Z$-scores decreased progressively during the course of therapy.

\section{Aortic Isthmus and Right Pulmonary Artery Diameter}

Changes in diameters and $Z$-scores of the aortic isthmus diameter were available from 13 of 14 fetuses with left heart malformations and of the right pulmonary artery from 1 fetus with Fallot tetralogy and poor pulmonary blood flow. In 11 of the fetuses with left heart malformations, marked diameter and $Z$-score increases of the aortic isthmus were observed (Fig. 4) during the course of therapy. In two fetuses, the diameter of the isthmus remained unchanged or decreased slightly such that their $Z$-scores decreased progressively during the course of therapy. In the fetus with Fallot tetralogy, marked diameter and Z-score increases of the right pulmonary artery were observed.

In fetuses in whom individual hypoplastic cardiovascular structures exhibited little or no increase in dimension during the course of chronic intermittent materno-fetal $\mathrm{HO}$, associated cardiac malformations-such as left superior caval vein-to-coronary sinus, unrestrictive ventricular septal defects, obstructed or stenotic atrioventricular valves, ventricular outflow obstruction, or semilunar valve stenosis-were observed (Table 1).

\section{Maternal and Fetal Safety}

Adverse maternal, fetal, or neonatal events during and after chronic intermittent materno-fetal HO were not observed during or after any of the treatment attempts. More specifically, neither detrimental changes to uterine and fetoplacental blood flows, fetal cardiac failure, nor preterm constriction of the ductus arteriosus or postnatal pulmonary hypertensive events occured in any of the fetuses. After $\mathrm{HO}$, the mothers exhibited normal chest X-rays and pulmonary function tests.

\section{Discussion}

This analysis found that chronic intermittent materno-fetal $\mathrm{HO}$ administered in late gestation may be associated with improvements of hypoplastic cardiovascular dimensions in fetuses with a variety of cardiac malformations. This finding may benefit postnatal treatment options and prognosis in suitable cases.

Reliable increases in hypoplastic cardiovascular dimensions during the course of chronic intermittent materno-fetal $\mathrm{HO}$ were observed in most fetuses with small ventricles that filled and emptied across normally built valves. These increases in the dimensions of cardiac

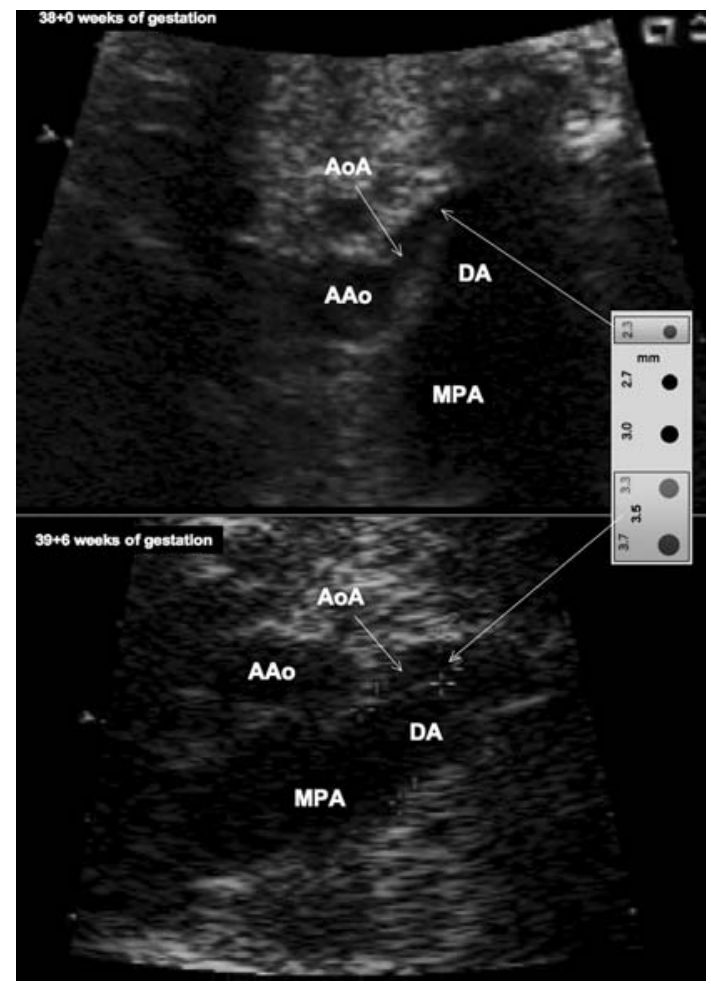

Fig. 5 Fetus with mild left heart hypoplasia and severe tubular hypoplasia of the transverse and distal aortic arch (top) at $38+0$ weeks of gestation (Table 1 , case no. 1). (Top panel) Note the sudden decrease in diameter from the ascending aorta (AAo) toward the transverse aortic arch (AoA). (Bottom panel) After 13 days of materno-fetal HO, the increase in left heart flow resulted in marked increases in the aortic isthmus diameter from $2.3 \mathrm{~mm}(-4.9 \mathrm{Z}$ score) to $3.5 \mathrm{~mm}(-2.7 \mathrm{Z}$-score). Ruler to the right of the figure is provided to facilitate the comprehension of these small dimensional changes on flow. $L A$ left atrium, $R A$ right atrium, $L V$ left ventricle, $R V$ right ventricle, $D A$ ductus arteriosus, $M P A$ main pulmonary artery; same scaling used for both great vessel images

structures were usually accompanied by increases in great vessel dimensions and even the aortic arch (Fig. 5). In contrast, the effect of materno-fetal $\mathrm{HO}$ was ameliorated or neutralized by the presence of unrestictive ventricular septal defects as well as obstructions to ventricular filling or emptying (e.g., left superior caval vein to the coronary sinus with absence of the left brachiocephalic vein, mitral valve stenosis, ventricular outflow tract obstruction, aortic valve stenosis).

Pending the absence of these ameliorating or neutralizing factors, the smaller a cardiovascular dimension was at the beginning of therapy, the larger its increase during the treatment course. In contrast, the more normal a cardiovascular dimension was at the beginning of materno-fetal $\mathrm{HO}$, the smaller its increase during the treatment course. The latter two observations may be explained by the fact that the increased volume load from materno-fetal HO must have a larger impact on smaller than on larger cardiovascular structures. 
The oxygen concentrations required to achieve marked fetal pulmonary vasodilation in this study were well below concentrations known to be harmful for adults, even when chronically administered. In contrast, little is known about fetal safety and the long-term effects of increasing fetal blood oxygen content by materno-fetal $\mathrm{HO}$ in late gestation. Therefore, careful observation of fetuses subjected to this novel treatment approach will be required not only before but also after birth.

Among the few data available, fetoscopic blood gas analysis in human fetuses between 17 and 22 weeks of gestation before and after materno-fetal $\mathrm{HO}$ with $100 \%$ oxygen showed that increases in fetal umbilical vein oxygen tension $\left(P_{\mathrm{O}_{2}}\right)$ from $39 \pm 4$ to $82 \pm 3 \mathrm{mmHg}$ and increases in oxygen saturation from $73 \% \pm 6 \%$ to $95 \% \pm 4 \%$ can be achieved by this strategy [4]. These fetal oxygen values resemble normal postnatal values for oxygen tension and saturation obtained in room air. However, because the maternal inspiratory oxygen concentration administered to achieve sufficient fetal pulmonary vasodilation and left heart growth in this study was approximately $45 \%$, it seems unlikely that oxygen tensions and concentrations that might be toxic to a mature fetus at appoximately 34 weeks of gestation can be achieved by this approach. Furthermore, detrimental changes in uterine and fetoplacental blood flows, cardiac failure, brain-sparing, or preterm constriction of the ductus arteriosus did not occur in these first cases.

My major concern after manipulation of fetal pulmonary circulation by materno-fetal $\mathrm{HO}$ so close to delivery was whether postnatal cardiopulmonary adaption in the first minutes of life would proceed normally and whether pulmonary hypertensive crises might occur in the coming hours and days. Fortunately, all fetuses adjusted normally to the postnatal environment without evidence for untoward pulmonary vascular events. However, until a much larger number of patients has been studied, neonates who were subjected to materno-fetal $\mathrm{HO}$ during fetal life should be carefully monitored for the occurence of pulmonary hypertension.

\section{Limitations of Study}

Given the variety and the small number of fetuses-most of which recruited under the umbrella of hypoplastic left heart complex-and the observation that the acute response as well as that over time of the pulmonary circulation to materno-fetal HO may differ depending on various anatomic factors (e.g., oval foramen closure), it was not possible to match the treatment group with an appropriate control group of fetuses that would have mirrored the spectrum of cardiac anomalies observed in the treated cases. I believe that sufficiently large numbers of cases with nearly identical lesions for a randomized trial protocol can only be provided in a timely fashion by multicenter efforts.

Alternatively, the dimensional changes from before to the end of materno-fetal $\mathrm{HO}$ in the treated fetuses were overlayed on growth charts derived from normal fetuses from the literature to provide some insight into the dimensional changes with time that are usually seen for the various structures. In addition, if one considers the rapid changes in cardiovascular dimensions that result from altered fetal hemodynamics from experiments of nature, such as twin-twin transfusion syndrome or vascular steal from large tumors, it becomes easier to conceive that similar changes might be achieved by iatrogenic manipulation of fetal cardiovascular flows employing oxygen.

Implications

The therapeutic potential of chronic intermittent maternofetal $\mathrm{HO}$ as a noninvasive tool for fetal cardiac intervention may now be tested in a large spectrum of prenatally detected fetal cardiac malformations with clinically relevant hypoplasia of cardiovascular structures. Apart from cases with hypoplastic left heart complex, many other cardiac malformations that are accompanied by severe underdevelopment of chambers, atrioventricular and semilunar valves, and great vessels (e.g., pumonary atresia with ventricular septal defect, double-inlet left ventricles, double-outlet right ventricles, balanced and unbalanced atrioventricular septal defects) might benefit from the method. In addition, improved left heart loading might benefit recovery of left ventricular function and growth after technically successful aortic balloon valvuloplasties. Given its simplicity, universal availability, and potential benefits for large numbers of patients, intensive research at dedicated centers is now required with the goals to optimize hyperoxygenation schedules, define all suitable cardiac malformations, and assess short and long-term safety of this novel therapeutic approach $[2,3]$.

Acknowledgments I greatly acknowledge the thoughtfulness of Kristina Tchatcheva and Rüdiger Stressig for recruiting most of the patients and also thank Ulrike Herberg for contributing most of the postnatal measurements. I also thank Rolf Fimmers for calculating the statistics. Last but not least, I am grateful to the Ronald McDonald Charity for donating the ultrasound system. The development of minimally invasive strategies and techniques for fetal cardiac interventions has over more than 15 years been supported by educational and research grants (Ko 1484/1-1, Ko 1484/2-1, Ko 1484/3-1, Ko 1484/3-2, Ko 1484/3-3) of the Deutsche Forschungsgemeinschaft (DFG), Bonn/Germany.

Open Access This article is distributed under the terms of the Creative Commons Attribution Noncommercial License which permits any noncommercial use, distribution, and reproduction in any medium, provided the original author(s) and source are credited. 


\section{References}

1. deAlmeida A, McQuinn T, Sedmera D (2007) Increased ventricular preload is compensated by myocyte proliferation in normal and hypoplastic fetal chick left ventricle. Circ Res 100:1363-1370

2. Kohl T, Tchatcheva K, Stressig R, Geipel A, Heitzer S, Gembruch $\mathrm{U}$ (2008) Maternal hyperoxygenation in late gestation promotes rapid increase of cardiac dimensions in fetuses with hypoplastic left hearts with intrinsically normal or slightly abnormal aortic and mitral valves [abstract]. Ultraschall Med 29:92

3. Kohl T (2009) Mending the tiniest hearts: an overview. In: Yagel S, Silverman NH, Gembruch U (eds) Fetal cardiology, 2nd edn. Informa Healthcare USA, New York, NY, pp 515-529

4. Mackenzie IZ, Castle B, Johnson P (1986) Blood gas analysis in the unstressed human fetus at 17-22 weeks' gestation. In: Rolfe P (ed) Fetal physiological measurements. Proceedings of the second international conference on fetal and neonatal physiological measurements, 1st edn. Butterworth, London, England, pp 156163
5. Matsui H, Mellander M, Roughton M, Jicinska H, Gardiner HM (2008) Morphological and physiological predictors of fetal aortic coarctation. Circulation 118:1793-1801

6. Mosteller RD (1987) Simplified calculation of body-surface area. N Engl J Med 317:1098

7. Pasquini L, Mellander M, Seale A, Matsui H, Roughton M, Ho SY et al (2007) Z-scores of the fetal aortic isthmus and duct: an aid to assessing arch hypoplasia. Ultrasound Obstet Gynecol 29:628-633

8. Rasanen J, Wood DC, Debbs RH, Cohen J, Weiner S, Huhta JC (1998) Reactivity of the human fetal pulmonary circulation to materno-fetal hyperoxygenation increases during the second half of pregnancy. A randomized study. Circulation 97:257-262

9. Schneider C, McCrindle BW, Carvalho JS, Hornberger LK, McCarthy KP, Daubeney PE (2005) Development of Z-scores from fetal cardiac dimensions from echocardiography. Ultrasound Obstet Gynecol 26:599-605 